properties of the 2D structures by measuring the specular reflection of light as a function of the wavelength of the incident field, observing a dip at $1100 \mathrm{~nm}$, corresponding to the third-order Bragg reflection band. The researchers also demonstrated the potential of the 2D array of $\mathrm{KTiOPO}_{4}$ rods to host an efficient nonlinear interaction by measurements of the diffracted light at the second-harmonic frequency of the incident wave.

This procedure enables control over the direction of growth relative to the orientation of the 2D structure. For any given application, it will then be possible to use the most appropriate combination of nonlinear or electro-optic coefficients of the material, something which is not always possible in bulk $\mathrm{KTiOPO}_{4}$, given the limited material birefringence. Such newly developed photonic crystals, which should be easily integrated in siliconbased devices, may find applications in generating light more efficiently at higher frequencies, in the electro-optic modulation of light, and in obtaining backward parametric amplification and oscillation.

\section{Submicrometer Technique Etches Curvilinear Silicon and Glass Patterns with HF-Saturated Hydrogel Stamps}

Applications in micro-optics, microfluidics, and microelectronics call for micrometer-sized devices with curvilinear or multilevel surface topographies. Casting elastomers against rigid masters is a parallel process that can rapidly create such architectures, but the resulting polymeric devices possess low resistance to mechanical wear, are permeable to gases, and often swell when exposed to organic solvents. Durable inorganic substrates, with better mechanical and chemical properties, may be patterned by expensive techniques such as reactive ion etching, laser ablation, or micromachining, but the throughput rate of these serial processes is low. In the July 16 issue of Advanced Materials (p. 2004; DOI: 10.1002/adma.200600716), followed by a recent publication in Chemistry of Materials (p. 4722; DOI: 10.1021) cm061468p), researchers at Northwestern University, led by B.A. Grzybowski, have presented a novel, inexpensive, directprinting technique that is suitable for rapid prototyping of multilevel reliefs in a variety of rigid substrates. The technique boasts a lateral resolution (in silicon) of several hundred nanometers, and it can pattern several square centimeters at a time.

Using soft lithographic techniques, Grzybowski and co-workers created a flexible hydrogel stamp by first patterning a reusable micropatterned master

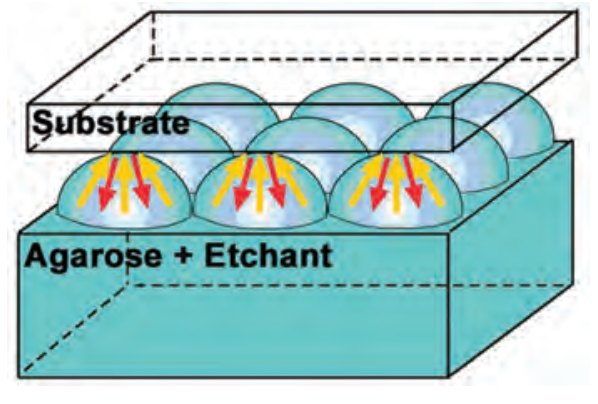

Figure 1. Schematic illustration of the experimental procedure for reactiondiffusion microetching.
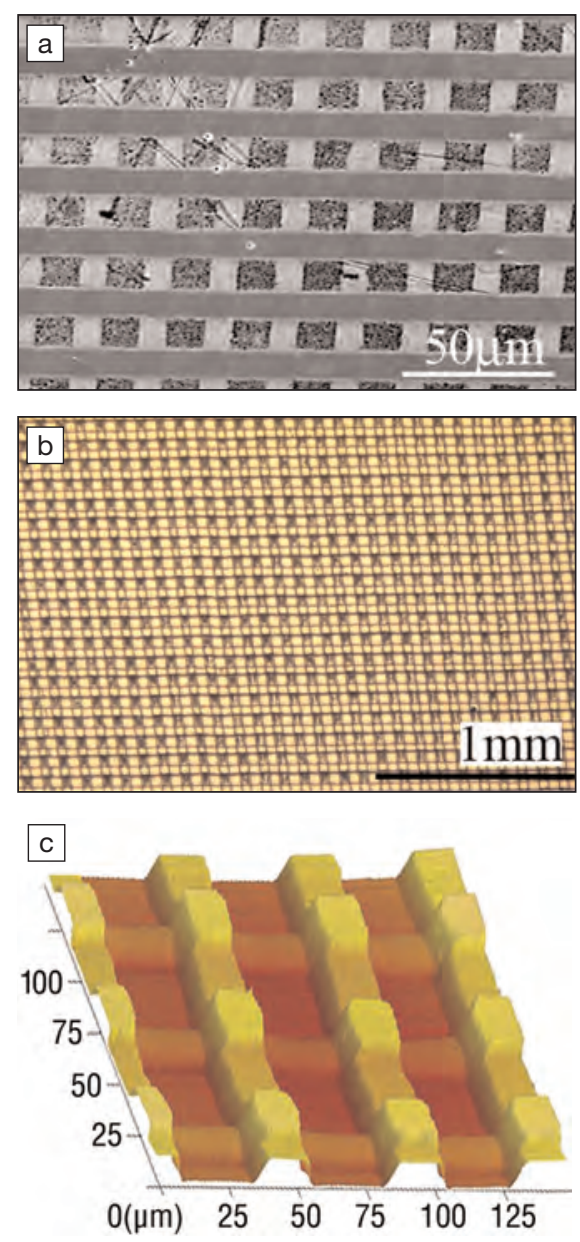

Figure 2. Three-dimensional (3D) architecture fabricated by sequential application of arrays of parallel lines along perpendicular directions. Pattern in GaAs: (a) scanning electron microscope image, (b) large-area optical micrograph, and (c) atomic force microscope image of a $3 D$ structure showing trenches 0.7 $\mu \mathrm{m}$ and $0.4 \mu \mathrm{m}$ deep. (Reprinted with permission from Chemistry of Materials 18 (20) (October 3, 2006) p. 4722; DOl: 10.1021/cm061468p; (C) 2006 American Chemical Society.) with the desired topography, then casting high-gel-strength agarose against it. They "inked" the agarose stamp by soaking it for $4 \mathrm{~h}$ in a $0.6 \mathrm{M}$ aqueous solution of hydrofluoric acid, with $0.1 \mathrm{vol} \%$ Triton X-100 surfactant.

The researchers laid the saturated stamp, feature side up, in light mineral oil, which confined the etchant to the raised features and reduced evaporation. Next, they placed the solid substrate to be patterned (e.g., a glass slide or a silicon wafer) directly upon the stamp, and ensured intimate contact by resting a small weight on top. Depending on the material system and the desired feature depth, the subsequent isotropic etching takes from several minutes to several days. The scientists then cleaned the etched surface using a piranha solution. The scheme is summarized in Figure 1.

The bulk agarose gel acts as a two-way "pump" that supplies fresh etchant and efficiently removes reaction products through diffusive transport. The etch rate is reaction-limited and is approximately constant. More concentrated acid solutions etch faster (which is helpful when making high-aspect-ratio features) but have detrimental effects on the stamp.

This easy-to-use, reliable method is reminiscent of woodcut printing, but on a submicrometer scale. The scientists have demonstrated the versatility of the technique by fabricating several difficult devices using their technique, including an array of convex/concave microlenses and a multilevel passive microfluidic mixer in glass.

"By exploiting other interfacial chemistries," Grzybowski said, "we have used the reaction-diffusion microetching process to pattern other materials, including zinc oxide and gallium arsenide." An example is shown in Figure 2.

RICH LOUIE

\section{Nanocrystalline, Flexible Photoelectrochromic Films Display Quick Response Time}

Changes in the optical properties of chromogenic materials occur by electric, thermal, or photo activation-for example, electrochromic "smart" windows that display switchable glazing. The change in color of photoelectrochromic (PEC) materials results from light absorption and application of an electric field. For example, a previously reported PEC device is based on an inorganic electrochromic film combined with a dye-sensitized semiconductor electrode. Recently, G. De Filpo and co-researchers at the Department of Chemistry, University of Calabria, Italy, developed a PEC film that uses a polymer- 
ic conductive support, thereby making the film flexible. In addition, the optical response is much faster than previous PEC devices due to an innovative assembly design.

As reported in a recent issue of Chemistry of Materials, De Filpo and co-researchers fabricated a nanocrystalline film by spincoating onto poly(ethylene terephthalate) a colloid containing $\mathrm{TiO}_{2}$ nanoparticles $25 \mathrm{~nm}$ in diameter. After hydrothermal treatment, the film was immersed in a phosphate buffer to attach $\mathrm{PO}_{4}^{-}$groups to the $\mathrm{TiO}_{2}$ nanospheres. The PET-TiO $-\mathrm{PO}_{4}$ films were then immersed in a solution containing methylene blue (MB), which adsorbed onto the film surface. It is well known that $\mathrm{MB}$ is photoactive in the triplet electronic state; photoreduction changes its color from blue to colorless. Oxidation of MB by means of application of direct current in the presence of an oxidative agent results in a color change from light blue to intense blue. Illumination with red light returns $\mathrm{MB}$ to its colorless, singlet ground state. A commercial microporous polar membrane (Nafion 112 from DuPont), which was previously immersed overnight in an electrolyte solution containing ethyl viologen diperchlorate (an oxidative agent), $0.2 \mathrm{M}$ triethylamine (an electron donor), and $0.5 \mathrm{M} \mathrm{LiClO}_{4}$ (a charge carrier), as well as a UV-photopolymerizable acrylate monomer and photoinitiator, was then placed between the $\mathrm{PET}-\mathrm{TiO}_{2}-\mathrm{PO}_{4}-\mathrm{MB}$ film and a PET film, and the entire device was irradiated with UV light for $5 \mathrm{~min}$ in order to polymerize the acrylate monomer, thereby linking the PEC film to the Nafion and PET supports. The device was sealed on all edges with cyanoacrylate glue.

When a voltage is applied to the nanocrystalline electrode, the bound MB molecules are oxidized and a fraction of the ethyl viologen molecules present in the electrolyte solution are reduced on the opposite electrode. This process is very fast and does not depend on the diffusion rate of the molecules toward the electrode. In addition, being able to transport electrical charges, the $\mathrm{TiO}_{2}$ nanoparticles have a very large surface area (about $50 \mathrm{~m}^{2} / \mathrm{g}$ ) so that it is possible to oxidize a large number of $\mathrm{MB}$ molecules per unit surface area. Using scanning electron microscopy, the cross section and surface morphology of the $\mathrm{TiO}_{2}$ layer were found to be very porous. The high mobility of the electrical charges results from the mutual contact of the $\mathrm{TiO}_{2}$ nanoparticles. Optical characterization of the device was performed with UV-vis spectroscopy, while the electro-optical response of the film was characterized by using two lasers simultaneously (He-Ne $632.8 \mathrm{~nm}, 1 \mathrm{~mW}$ and $5 \mathrm{~mW}$ ). A lowerpowered reading laser was used to measure the optical transmission of the film, while a higher-powered writing laser was used to promote the bleaching process. Before being exposed to red light, the device displayed an absorption maximum at $660 \mathrm{~nm}-$ characteristic of $\mathrm{MB}$ - resulting in an intense blue color and an optical transmission of $1 \%$. The device becomes transparent (an optical transmission of 57\%) and the absorption maximum vanishes $20 \mathrm{~s}$ after irradiation with red light. However, a 1-s electrical impulse of $0.4 \mathrm{~V}$ dc results in re-coloring the device in about $600 \mathrm{~ms}$.

De Filpo said that future work is planned to improve the bleaching time of the film, which is still too long for practical use. However, the flexible nature of the device expands its use beyond traditional applications.

STEVEN TROHALAKI

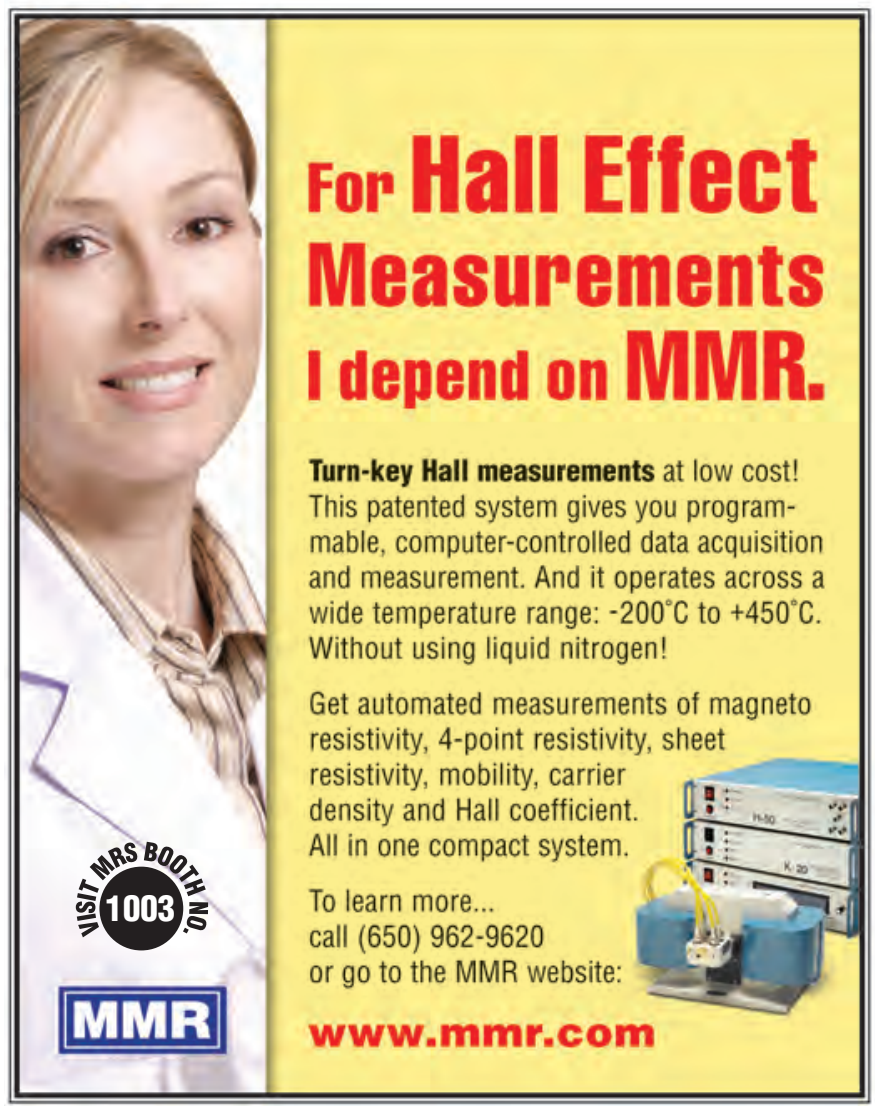

For more information, see http://www.mrs.org/bulletin_ads

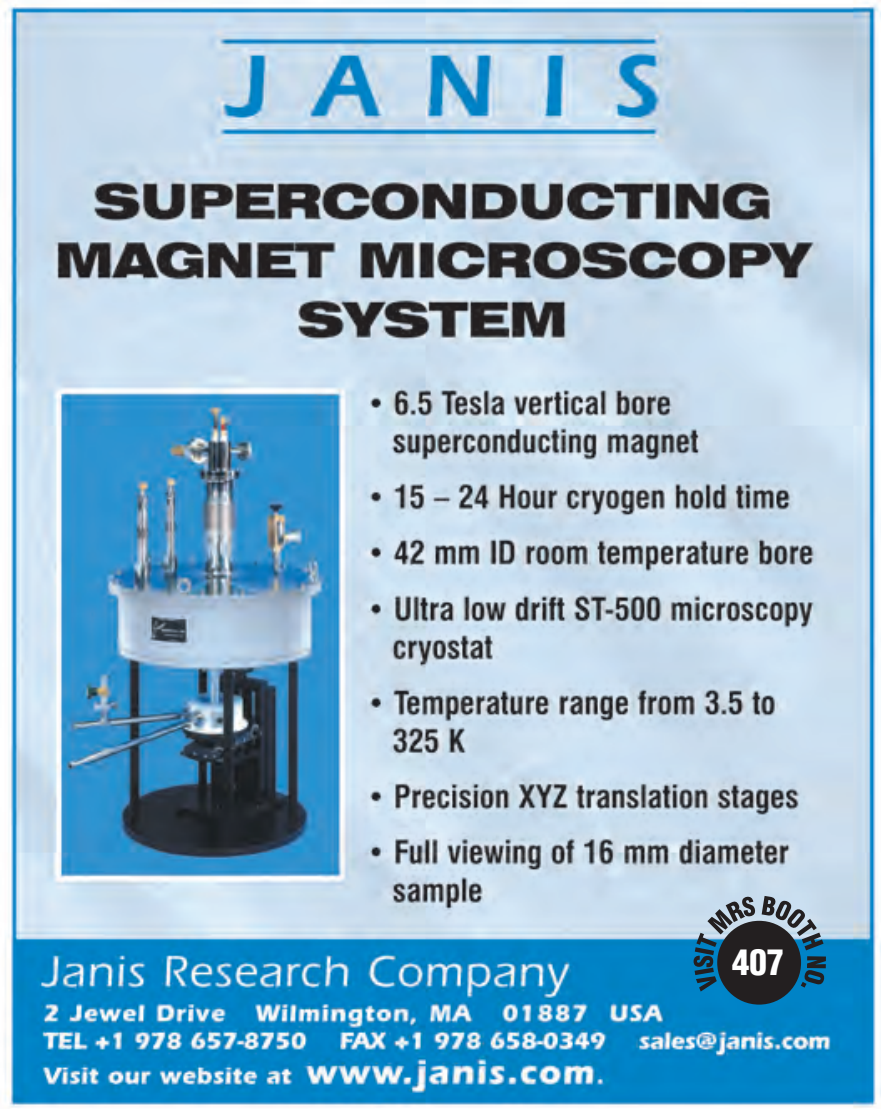

For more information, see http://www.mrs.org/bulletin_ads 